PROCEEDINGS OF THE

AMERICAN MATHEMATICAL SOCIETY

Volume 128, Number 7 , Pages 1963-1970

S 0002-9939(99)05197-7

Article electronically published on November 1, 1999

\title{
GROWTH PROPERTIES OF SUPERHARMONIC FUNCTIONS ALONG RAYS
}

\author{
STEPHEN J. GARDINER
}

(Communicated by Albert Baernstein II)

\begin{abstract}
This paper gives a precise topological description of the set of rays along which a superharmonic function on $\mathbb{R}^{n}$ may grow quickly. The corollary that arbitrary growth cannot occur along all rays answers a question posed by Armitage.
\end{abstract}

\section{InTRODUCTION}

The purpose of this paper is to show that a result of Armitage (Theorem $\mathrm{A}$ below) about the growth along rays of superharmonic functions on $\mathbb{R}^{2}$ does indeed, contrary to what is suggested by the observations in [1], have a natural (but rather non-obvious) generalization to higher dimensions. As a corollary we solve a problem posed in [1] by showing that a superharmonic function on $\mathbb{R}^{n}$ cannot grow too quickly on a topologically large set of rays. We begin by recording the following [1] Theorem 2].

Theorem A. Let $E \subseteq[0,2 \pi)$ and let $u$ be a superharmonic function on $\mathbb{R}^{2}$. If

$$
\liminf _{r \rightarrow+\infty} u\left(r e^{i \theta}\right)>-\infty \quad(\theta \in E)
$$

and, for each positive number $\mu$, the closure of the set

$$
\left\{\theta \in[0,2 \pi): r^{-\mu} u\left(r e^{i \theta}\right) \rightarrow+\infty \text { as } r \rightarrow+\infty\right\}
$$

contains $E$, then $E$ is of first category.

However, it was remarked in [1] that the natural analogue of Theorem $\mathrm{A}$ in higher dimensions fails because of the following fact. We denote by $S$ the unit sphere in $\mathbb{R}^{n}$.

Theorem B. Let $n \geq 3$ and let $M:[0,+\infty) \rightarrow(0,+\infty)$ be increasing. There exists a subset $E$ of $S$ and a superharmonic function $u$ on $\mathbb{R}^{n}$ such that

$$
u(r y) / M(r) \rightarrow+\infty \quad(r \rightarrow+\infty ; y \in E)
$$

and $S \backslash E$ is of first category in $S$.

It was even shown in [1, Proposition 2] that the set $E$ in Theorem $B$ can have full surface area measure, and this led to the question [1, p. 251] of whether it is possible to have $E=S$. We will show below that Theorem $\mathrm{A}$ does have a natural

Received by the editors April 1, 1998 and, in revised form, August 13, 1998.

2000 Mathematics Subject Classification. Primary 31B05.

(C)2000 American Mathematical Society 
generalization to higher dimensions, and will observe as an immediate consequence that $E$ cannot equal $S$ in Theorem B. First we make some definitions.

The fine topology on $\mathbb{R}^{n}(n \geq 1)$ is the coarsest topology for which all superharmonic functions are continuous. (See Doob [3, 1.XI] for an account of this topology and the associated notion of thinness of a set.) It has a natural extension to compactified space $\mathbb{R}^{n} \cup\{\infty\}$ (see [3, 1.XI.5]). From now on we will assume that $n \geq 2$. By the fine topology on $S$ we mean the topology induced on $S$ by the fine topology on $\mathbb{R}^{n-1} \cup\{\infty\}$ under the stereographic projection $f: \mathbb{R}^{n-1} \cup\{\infty\} \rightarrow S$ given by

$$
f\left(x^{\prime}\right)= \begin{cases}\left(1+\left|x^{\prime}\right|^{2}\right)^{-1}\left(2 x_{1}, \ldots, 2 x_{n-1},\left|x^{\prime}\right|^{2}-1\right) & \left(x^{\prime}=\left(x_{1}, \ldots, x_{n-1}\right)\right), \\ (0, \ldots, 0,1) & \left(x^{\prime}=\infty\right)\end{cases}
$$

where $\left|x^{\prime}\right|=\left(x_{1}^{2}+\cdots+x_{n-1}^{2}\right)^{1 / 2}$. When $n=2$ the fine topology on $S$ coincides with the Euclidean one since the superharmonic functions on $\mathbb{R}$ are precisely the concave functions, and hence are already continuous. The fine topology on $\mathbb{R}^{n}$ has the Baire property, so the same is true of the fine topology on $S$. When $n \geq 3$, a subset $A$ of $S$ will be called polar in $S$ if $A \backslash\{(0, \ldots, 0,1)\}$ is the stereographic image of a polar set in $\mathbb{R}^{n-1}$. This is equivalent (cf. [10, Chapter II, $\left.\S \S 3,4\right]$ ) to $A$ having zero capacity with respect to the kernel $|x-y|^{3-n}(n \geq 4)$ or $\log 1 /|x-y|$ $(n=3)$. When $n=2$, only the empty set will be called polar in $S$. Our main result is as follows.

Theorem 1. Let $E \subseteq S$ and $n \geq 2$. The following are equivalent:

(a) there is a superharmonic function $u$ on $\mathbb{R}^{n}$ such that

$$
\liminf _{r \rightarrow+\infty} u(r y)>-\infty \quad(y \in E)
$$

and such that, for each positive number $\mu$ and each finely open subset $U$ of $S$ for which $U \cap E$ is non-polar in $S$, the set

$$
\left\{y \in U: r^{-\mu} u(r y) \rightarrow+\infty \text { as } r \rightarrow+\infty\right\}
$$

is non-polar in $S$;

(b) there is a superharmonic function $u$ on $\mathbb{R}^{n}$ such that, for each positive number $\mu$,

$$
r^{-\mu} u(r y) \rightarrow+\infty \quad(r \rightarrow+\infty ; y \in E)
$$

(c) for each increasing function $M:[0,+\infty) \rightarrow(0,+\infty)$ there is a superharmonic function $u$ on $\mathbb{R}^{n}$ such that

$$
u(r y) / M(r) \rightarrow+\infty \quad(r \rightarrow+\infty ; y \in E)
$$

(d) $E$ is of first category with respect to the fine topology on $S$.

Corollary 1. There is no superharmonic function $u$ on $\mathbb{R}^{n}$ such that $r^{-\mu} u(r y) \rightarrow$ $+\infty$ as $r \rightarrow+\infty$ for each $y$ in $S$ and each positive number $\mu$.

When $n=2$, the implication (a) $\Rightarrow$ (d) of Theorem 1 corresponds to Theorem A since we can delete "finely" and "fine", and replace "non-polar in $S$ " by "nonempty". In higher dimensions this implication is more delicate and most of the proof of Theorem 1 is devoted to it. Corollary 1 answers negatively the question of Armitage mentioned above.

Following some preliminary lemmas in $\$ 2$ Theorem 1 is proved in 33 , The arguments involve modifications of ideas from [7] and [4] together with some new ingredients. We note from [4, Proposition 1] that there is no implication in either 
direction between the notions of first category with respect to the Euclidean and fine topologies on $S$.

\section{Preliminary Lemmas}

2.1. We refer to [7, Lemma 2] or [8] for a proof of the following lemma.

Lemma A. Let $n \geq 3$ and $A^{\prime} \subseteq \mathbb{R}^{n-1}$, let $x^{\prime} \in \mathbb{R}^{n-1}$ and $t \in \mathbb{R}$. Then $A^{\prime}$ is thin at $x^{\prime}$ in $\mathbb{R}^{n-1}$ if and only if $A^{\prime} \times \mathbb{R}$ is thin at $\left(x^{\prime}, t\right)$ in $\mathbb{R}^{n}$.

A subset $A$ of $S$ will be called $S$-thin at a point $z$ of $S$ if $z$ is not a limit point of $A$ with respect to the fine topology on $S$.

Lemma 1. Let $n \geq 3$, let $A \subseteq S$ and $C=\{r y: y \in A$ and $r>0\}$, let $z \in S$ and $\rho>0$. Then $C$ is thin at $\rho z$ if and only if $A$ is $S$-thin at $z$. Also, $C$ is polar in $\mathbb{R}^{n}$ if and only if $A$ is polar in $S$.

In proving Lemma 1 we may assume that $\rho=1$, since thinness is invariant under dilation. Let $A^{\prime}$ (resp. $z^{\prime}$ ) denote the pre-image of $A$ (resp. $z$ ) under the stereographic projection. Then, by inverting $A^{\prime}$ in the unit sphere of $\mathbb{R}^{n-1}$, we see that $A$ is $S$-thin at $(0, \ldots, 0,1)$ if and only if the reflection of $A$ in $\mathbb{R}^{n-1} \times(0,+\infty)$ is $S$-thin at $(0, \ldots, 0,-1)$. Since thinness in $\mathbb{R}^{n}$ is preserved by reflection, we may assume from now on that $z \neq(0, \ldots, 0,1)$. Further, since thinness is a local property, we may assume that $(0, \ldots, 0,1) \notin \bar{A}$.

By definition $A$ is $S$-thin at $z$ if and only if $A^{\prime}$ is thin at $z^{\prime}$ in $\mathbb{R}^{n-1}$, which is equivalent, by Lemma 1 , to the condition that $A^{\prime} \times(-1,1)$ is thin at $\left(z^{\prime}, 0\right)$ in $\mathbb{R}^{n}$. Since the mapping

$$
\left(x^{\prime}, x_{n}\right) \mapsto \exp \left(x_{n}\right) f\left(x^{\prime}\right),
$$

from $\mathbb{R}^{n}$ to $\mathbb{R}^{n} \backslash\{0\}$, is smooth and has a smooth inverse, it is routine to check that $A^{\prime} \times(-1,1)$ is thin at $\left(z^{\prime}, 0\right)$ if and only if the set

$$
\left\{r y: y \in A \text { and } e^{-1}<r<e\right\}
$$

is thin at $z$. (See [9, Theorem 10.6] for an argument of this type.) This latter condition is equivalent to the thinness of $C$ at $z$, again because thinness is a local property.

The final assertion of Lemma 1 follows from the characterization of polar sets in $\mathbb{R}^{n}$ as sets which are everywhere thin.

2.2. If $E \subseteq \mathbb{R}^{n}$ and $r>0$, then we define $r E=\{r y: y \in E\}$.

Lemma 2. Let $n \geq 3$, let $U \subseteq S$ be open with respect to the fine topology on $S$ and let $C=\{r y: y \in U$ and $r>0\}$. Then the fine components of $C$ are of the form $D=\{r y: y \in V$ and $r>0\}$, where $V$ is open and connected with respect to the fine topology on $S$.

To prove this, we first observe that, if $\Omega$ is a finely open set in $\mathbb{R}^{n}$, then there exists $E \subseteq S$, polar as a subset of $\mathbb{R}^{n}$, such that the set $\{r>0: r y \in \Omega\}$ is open in $\mathbb{R}$ whenever $y \in S \backslash E$. This can be established using an argument similar to that employed to prove the analogous statement with parallel lines in place of rays (see [4, Corollary 1(i)]).

Now let $U$ and $C$ be as in the statement of Lemma 2 It follows from Lemma 1 that $C$ is finely open in $\mathbb{R}^{n}$. Let $D$ be a fine component of $C$. Then $D$ is also 
finely open by the local connectedness of the fine topology (see [5] p. 92]). By the observation in the preceding paragraph there exists $\kappa>1$ such that

$$
(r D) \cap D \neq \varnothing \quad\left(\kappa^{-1}<r<\kappa\right) .
$$

Since $r D$ is also a fine component of the dilation-invariant set $C$, it follows that $r D=D$ whenever $\kappa^{-1}<r<\kappa$. Repeated application of this fact leads to the conclusion that $r D=D$ for all $r>0$. Hence $D$ is of the form $\{r y: y \in V$ and $r>0\}$, and it follows from Lemma 1 again that $V$ is finely open in $S$. Finally, it is clear from the fine connectedness of $D$ that $V$ must be finely connected in $S$.

\section{Proof of Theorem 1}

3.1. We begin by proving that (d) implies (c). For this we need the following (see [6, Theorem 3.19 and Corollary 3.21] or [1] for more general results of this type, and [6, §3.2] for a discussion of local connectedness in this context).

Theorem C. Let $F$ be a (Euclidean) closed set in $\mathbb{R}^{n}$ such that $\left(\mathbb{R}^{n} \cup\{\infty\}\right) \backslash F$ is connected and locally connected and such that the fine interior of $F$ is empty. Then, for any continuous function $u$ on $F$ and any positive number $\varepsilon$, there exists a harmonic function $v$ on $\mathbb{R}^{n}$ such that $|v-u|<\varepsilon$ on $F$. (If $n=2$, then the word "fine" can be omitted.)

Now suppose that condition (d) holds and that $n \geq 3$. Then there is an increasing sequence $\left(K_{k}\right)$ of (Euclidean) closed subsets of $S$ which have empty interiors with respect to the fine topology on $S$, and a set $Z$ which is polar in $S$, such that $E \subseteq\left(\bigcup_{k} K_{k}\right) \cup Z$ (see [4] Lemma 2]). The set

$$
Y=\{r z: z \in Z \text { and } r>0\}
$$

is polar in $\mathbb{R}^{n}$, by Lemma 1 so there is a positive superharmonic function $s$ on $\mathbb{R}^{n}$ such that $s=+\infty$ on $Y$. Given an increasing function $M:[0,+\infty) \rightarrow(0,+\infty)$, let $g:[0,+\infty) \rightarrow(0,+\infty)$ be a continuous function such that

$$
g(r) \geq 1+r M(r) \quad(r \geq 0)
$$

and let

$$
F=\bigcup_{k=1}^{\infty}\left\{r y: y \in K_{k} \text { and } r \geq k\right\} .
$$

Then $F$ is a (Euclidean) closed set and, since the sets $K_{k}$ are nowhere (Euclidean) dense in $S$, the set $\left(\mathbb{R}^{n} \cup\{\infty\}\right) \backslash F$ is connected and locally connected. In fact, the sets $K_{k}$ are nowhere dense with respect to the fine topology on $S$ and so, by

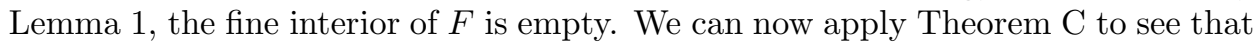
there is a harmonic function $v$ on $\mathbb{R}^{n}$ such that

$$
|v(x)-g(|x|)|<1 \quad(x \in F) .
$$

The function $u=v+s$ is thus a superharmonic function on $\mathbb{R}^{n}$ which satisfies

$$
u(x)>g(|x|)-1 \geq|x| M(|x|) \quad(x \in F)
$$

and $u=+\infty$ on $Y$. Hence condition (c) of Theorem 1 holds.

If $n=2$ and condition (d) holds, then there is an increasing sequence $\left(K_{k}\right)$ of closed nowhere dense subsets of $S$ such that $E \subseteq \bigcup_{k} K_{k}$, and we can again use Theorem $\mathrm{C}$ to see that condition (c) follows. 
3.2. It is clear that $(\mathrm{c}) \Rightarrow(\mathrm{b}) \Rightarrow(\mathrm{a})$, so it remains to show that $(\mathrm{a}) \Rightarrow(\mathrm{d})$. The case where $n=2$ is covered by Theorem $\mathrm{A}$ so we will assume for the remainder of this section that $n \geq 3$.

Suppose that (a) holds but (d) fails, and let

$$
E_{k}=\{y \in E: u(r y)>1-k \text { for all } r>0\} \quad(k \in \mathbb{N}) .
$$

Then $\bigcup_{k} E_{k}=E$ by (1), so there exists $k_{0}$ in $\mathbb{N}$ such that the closure of $E_{k_{0}}$ with respect to the fine topology on $S$ has non-empty fine interior $U$. By Lemma 2 there is a non-empty finely open subset $V$ of $U$ such that the set

$$
D=\{r y: y \in V \text { and } r>0\}
$$

is finely connected in $\mathbb{R}^{n}$. From Lemma 1 and the fine continuity of $u$ it follows that

$$
u \geq 1-k_{0}>-k_{0} \text { on } D .
$$

The set $V \cap E$ is non-polar in $S$, so the same is true, by hypothesis, of the sets

$$
V_{m}=\left\{y \in V: r^{-m} u(r y) \rightarrow+\infty \text { as } r \rightarrow+\infty\right\} \quad(m \in \mathbb{N}) .
$$

Let

$$
V_{m, l}=\left\{y \in V: r^{-m} u(r y) \geq 1 \text { whenever } r \geq 2^{l}\right\} \quad(m, l \in \mathbb{N}) .
$$

Then $V_{m} \subseteq \bigcup_{l} V_{m, l}$. For each $m$ in $\mathbb{N}$ we choose $l(m)$ such that $V_{m, l(m)}$ is non-polar in $S$. Let

$$
D_{m}=\left\{r y: y \in V_{m, l(m)} \text { and } r \geq 1\right\}
$$

and let $v_{m}$ denote the fine balayage of the constant function 1 relative to the set $D_{m}$ in $D$. (We refer to Fuglede [5] for concepts related to finely superharmonic functions.) By Lemma 1 the set $D_{m}$ is non-polar in $\mathbb{R}^{n}$, so $v_{m}>0$ in $D$.

Let

$$
F_{m, j}=\left\{x \in V: v_{m}(x)>1 / j\right\} \quad(m, j \in \mathbb{N}) .
$$

Then $\bigcup_{j} F_{m, j}=V$ for each $m$. Let $0<\varepsilon<\mathcal{C}(V)$ where $\mathcal{C}(\cdot)$ denotes Newtonian capacity in $\mathbb{R}^{n}$. We choose $j(1)$ such that

$$
\mathcal{C}\left(F_{1, j(1)}\right)>\varepsilon
$$

and then a compact subset $K_{1}$ of $F_{1, j(1)}$ such that $\mathcal{C}\left(K_{1}\right)>\varepsilon$. We proceed inductively: given a compact subset $K_{m}$ of $V$ such that $\mathcal{C}\left(K_{m}\right)>\varepsilon$, we choose $j(m+1)$ such that $\mathcal{C}\left(K_{m} \cap F_{m+1, j(m+1)}\right)>\varepsilon$, and then a compact subset $K_{m+1}$ of $K_{m} \cap F_{m+1, j(m+1)}$ such that $\mathcal{C}\left(K_{m+1}\right)>\varepsilon$. We define the compact set $K=\bigcap_{m} K_{m}$. Then $K$ is non-polar in $\mathbb{R}^{n}$ and $K \subseteq F_{m, j(m)}$ for all $m$. Let $v$ denote the fine balayage of the constant function 1 relative to $K$ in $D$ and let

$$
F_{j}=\left\{x \in V: v(x)>j^{-1}\right\} \quad(j \in \mathbb{N}) .
$$

Then $v>0$ and $\bigcup_{j} F_{j}=V$, so there exists $j_{0}$ such that the set $F_{j_{0}}$, which is finely open in $S$, is non-empty.

If $A \subseteq \mathbb{R}^{n}$ and $w$ is a positive superharmonic function on $\mathbb{R}^{n}$, then let $\widehat{R}_{w}^{A}$ denote the balayage of $w$ relative to $A$ with respect to superharmonic functions on $\mathbb{R}^{n}$. We 
define

$$
g(x, y)=|x-y|^{2-n}-\widehat{R}_{|\cdot-y|^{2-n}}^{\mathbb{R}^{n} \backslash D}(x) \quad\left(x, y \in \mathbb{R}^{n} ; x \neq y\right),
$$

and note that $g(x, \cdot)$ is subharmonic on $\mathbb{R}^{n} \backslash\{x\}$ for each $x$, and $g(\cdot, y)$ is subharmonic on $\mathbb{R}^{n} \backslash\{y\}$ for each $y$. (See [3, 1.X.3] for the symmetry of $g(\cdot, \cdot)$.) Since $g(\cdot, \cdot)$ is locally bounded above on

$$
\{r y: y \in S \text { and } r>3 / 2\} \times\{r y: y \in S \text { and } 0<r<3 / 2\},
$$

a result of Avanissian [2, Théorème 9] now asserts that $g(\cdot, \cdot)$ is subharmonic on this subset of $\mathbb{R}^{2 n}$. In particular, $g$ is upper semicontinuous on the set $(2 S) \times S$ (recall the definition of $r E$ in $\$ 2.2)$. Let $F$ be a compact subset of $F_{j_{0}}$ with non-empty fine interior and let

$$
L_{i}=\left\{(y, z) \in F^{2}: g(2 y, z) \geq i^{-1} \text { and } g(y, 2 z) \geq i^{-1}\right\} \quad(i \in \mathbb{N}) .
$$

Then $\bigcup_{i} L_{i}=F^{2}$, since when $y, z \in D$, the non-negative functions $g(y, \cdot)$ and $g(\cdot, z)$ are finely superharmonic and not identically zero on the fine domain $D$, and hence are strictly positive there. The product topology on $S^{2}$, generated from the fine topology on $S$, also has the Baire property since it has a neighbourhood base of Euclidean compact sets (cf. [3. pp. 167, 168]). Hence there exists $i_{0}$ in $\mathbb{N}$ such that the compact set $L_{i_{0}}$ has non-empty interior with respect to this product topology. In particular, there exist compact subsets $M_{1}$ and $M_{2}$ of $F_{j_{0}}$ which have non-empty interiors with respect to the fine topology on $S$ and which satisfy

$$
g(2 y, z) \geq i_{0}^{-1} \quad \text { and } \quad g(y, 2 z) \geq i_{0}^{-1} \quad\left(y \in M_{1}, z \in M_{2}\right) .
$$

The sets $M_{1}$ and $M_{2}$ have positive $(n-1)$-dimensional measure and hence are non-polar in $\mathbb{R}^{n}$. We define $c=\min \left\{\mathcal{C}\left(M_{1}\right), \mathcal{C}\left(M_{2}\right)\right\}$ and choose $m_{1}$ such that $2^{m_{1}}>i_{0} / c$.

Now let $G_{\Omega}(\cdot, \cdot)$ denote the Green function of the open set

$$
\Omega=\left\{x \in \mathbb{R}^{n}: u(x)+k_{0}>0\right\}
$$

and let $l \geq l\left(m_{1}\right)$. (For the definition of $l\left(m_{1}\right)$ see the line following (3).) Then $D \subseteq \Omega$ by (2), and since $u\left(2^{l} x\right) \geq 2^{l m_{1}}$ when $x \in D_{m_{1}}$ (see (3) and (4)), it follows that

$$
u\left(2^{l} x\right)+k_{0} \geq 2^{l m_{1}} v_{m_{1}}(x) \quad(x \in D)
$$

(see the line following (4) for the definition of $v_{m_{1}}$ ). Thus

$$
u\left(2^{l} x\right)+k_{0} \geq \frac{2^{l m_{1}}}{j\left(m_{1}\right)} \quad\left(x \in F_{m_{1}, j\left(m_{1}\right)}\right)
$$

in view of (5), so

$$
u\left(2^{l} x\right)+k_{0} \geq \frac{2^{l m_{1}}}{j\left(m_{1}\right)} v(x) \quad(x \in D),
$$

since $K \subseteq F_{m_{1}, j\left(m_{1}\right)}$. Hence

$$
u\left(2^{l} x\right)+k_{0} \geq \frac{2^{l m_{1}}}{j_{0} j\left(m_{1}\right)} \quad\left(x \in F_{j_{0}}\right)
$$

in view of (6). In particular, the above inequality holds for all $x$ in $M_{1} \cup M_{2}$. 
Thus

$$
u(x)+k_{0} \geq \frac{2^{l m_{1}}}{j_{0} j\left(m_{1}\right)} w_{i, l}(x) \quad(x \in \Omega ; i=1,2),
$$

where $w_{i, l}$ denotes the balayage of the constant function 1 relative to the set $2^{l} M_{i}$ with respect to positive superharmonic functions on $\Omega$. Each function $w_{i, l}$ is the potential on $\Omega$ of a measure $\mu_{i, l}$ with support in the compact set $2^{l} M_{i}$ and

$$
\mu_{i, l}\left(2^{l} M_{i}\right) \geq 2^{l(n-2)} c,
$$

using the fact that $G_{\Omega}(x, y) \leq|x-y|^{2-n}$ and the standard dilation property of Newtonian capacity. Similarly

$$
g(r x, r y)=r^{2-n} g(x, y) \quad\left(x, y \in \mathbb{R}^{n} ; r>0\right) .
$$

Since

$$
g(x, y) \leq|x-y|^{2-n}-\widehat{R}_{|\cdot-y|^{2-n}}^{\mathbb{R}^{n} \backslash \Omega}(x)=G_{\Omega}(x, y) \quad\left(x, y \in \mathbb{R}^{n} ; x \neq y\right),
$$

we see from (7), (9) and (10) that

$$
\begin{aligned}
w_{i, l}(x) & =\int_{2^{l} M_{i}} G_{\Omega}(x, y) d \mu_{i, l}(y) \\
& \geq \int_{2^{l} M_{i}} g(x, y) d \mu_{i, l}(y) \\
& \geq 2^{(l-1)(2-n)} i_{0}^{-1} \mu_{i, l}\left(2^{l} M_{i}\right) \\
& \geq c / i_{0} \quad\left(x \in 2^{l-1} M_{3-i} ; i=1,2\right) .
\end{aligned}
$$

Thus, by (8),

$$
u(x)+k_{0} \geq\left(c / i_{0}\right) 2^{l m_{1}}\left\{j_{0} j\left(m_{1}\right)\right\}^{-1} \quad\left(x \in 2^{l-1}\left(M_{1} \cup M_{2}\right)\right),
$$

and repeated application of this argument yields

$$
u(x)+k_{0} \geq\left(c / i_{0}\right)^{l} 2^{l m_{1}}\left\{j_{0} j\left(m_{1}\right)\right\}^{-1} \quad\left(x \in M_{1} \cup M_{2}\right) .
$$

Since $m_{1}$ was chosen such that $2^{m_{1}}>i_{0} / c$, and $l$ can be arbitrarily large, we obtain the contradictory conclusion that $u=+\infty$ on the non-polar set $M_{1} \cup M_{2}$. Thus condition (d) of Theorem [1] must hold and the proof is complete.

Corollary 1 follows immediately.

\section{REFERENCES}

1. D. H. Armitage, Radial limits of superharmonic functions in the plane, Colloq. Math. 67 (1994), 245-252. MR 96a:31001

2. V. Avanissian, Fonctions plurisousharmoniques et fonctions doublement sousharmoniques, Ann. Sci. École Norm. Sup. (3) 78 (1961), 101-161. MR 24:A2052

3. J. L. Doob, Classical potential theory and its probabilistic counterpart, Springer, New York, 1984. MR 85k:31001

4. M. R. Essén and S. J. Gardiner, Limits along parallel lines and the classical fine topology, J. London Math. Soc., to appear.

5. B. Fuglede, Finely harmonic functions, Lecture Notes in Math. 289, Springer, Berlin, 1972. MR 56:8883

6. S. J. Gardiner, Harmonic approximation, LMS Lecture Note Series 221, Cambridge Univ. Press, 1995. MR 96j:31001 
7. S. J. Gardiner, The Lusin-Privalov theorem for subharmonic functions, Proc. Amer. Math. Soc. 124 (1996), 3721-3727. MR 97m:31003

8. W. Hansen, Abbildungen harmonischer Räume mit Anwendung auf die Laplace und Wärmeleitungsgleichung, Ann. Inst. Fourier Grenoble 21, 3 (1971), 203-216. MR 49:617

9. L. L. Helms, Introduction to potential theory, Krieger, New York, 1975. MR 57:659

10. N. S. Landkof, Foundations of modern potential theory, Springer, Berlin, 1972. MR 50:2520

11. A. A. Shaginyan, Uniform and tangential harmonic approximation of continuous functions on arbitrary sets, Mat. Zametki 9 (1971), 131-142; English translation in Math. Notes 9 (1971), $78-84$.

Department of Mathematics, University College Dublin, Dublin 4, Ireland

E-mail address: stephen.gardiner@ucd.ie 